\title{
Power Optimisation by Mass Tuning for MEMS Piezoelectric Cantilever Vibration Energy Harvesting
}

\author{
Yu Jia, Member, IEEE and Ashwin A. Seshia Senior Member, IEEE \\ Nanoscience Centre, University of Cambridge, Cambridge CB3 OFF, UK \\ E-mail: yu.jia.gb@ieee.org, aas41@cam.ac.uk
}

Draft: October 26, 2015

\begin{abstract}
A cantilever with an end mass is one of the most popular designs for piezoelectric MEMS vibration energy harvesting. The inclusion of a proof mass near the free end of a micro-cantilever can significantly enhances the power responsiveness of a vibration energy harvester per unit acceleration. However, the accommodation of the proof mass comes at the expense of the active piezoelectric area. This paper numerically and experimentally investigates this compromise and explores the optimal proof-mass-to-cantilever-length ratio for power maximisation. It was found that an end mass occupying about $\mathbf{6 0} \%$ to $\mathbf{7 0} \%$ of the total cantilever length is optimal within linear response, and they notably outperform comparable cantilevers with $\mathbf{4 0} \%$ and $\mathbf{5 0} \%$ of end mass. Additionally, nonlinear squeeze film air damping within the chip package was found to adversely affect the cantilevers with larger mass more significantly. A harvester prototype with $\mathbf{7 0} \%$ of the length covered by end mass $\left(5.0 \mathrm{~mm}^{3}\right)$ was able to generate $1.78 \mu \mathrm{W}$ at $0.6 \mathrm{~ms}^{-2}$ and up to $20.5 \mu \mathrm{W}$ at $2.7 \mathrm{~ms}^{-2}$ and $210 \mathrm{~Hz}$ when not limited by nonlinear damping. This result outperforms the previously reported counterparts in the literature by nearly an order of a magnitude in terms of power density normalised against acceleration squared.
\end{abstract}

\section{Introduction}

Vibration energy harvesting (VEH) aims to realise net-zero-power micro-systems, which can potentially complement other emerging technologies such as wireless sensors and implantable medical devices. Within the micro-electromechanical system (MEMS) VEH space, piezoelectric transducers have generally performed more favourably than their electrostatic counterparts in terms of power density, while electromagnetic transducers do not scale down well [1].

Additionally, piezoelectric materials such as alu- minium nitride $(\mathrm{AlN})$ and zinc oxide $(\mathrm{ZnO})$ are relatively more compatible with MEMS fabrication than electromagnetic generators [2]. More recently, bulk piezoelectric materials such as lead zirconate titanate (PZT) has also seen increasing integration with MEMS fabrication processes [3, 4].

While progress has been made to fabricate MEMS PZT from powder or solgel $[5,6]$, the process is yet to be perfected and the quality of the produced PZT has yet to demonstrate itself to the standard of its bulk counterpart. Therefore, despite the promising piezoelectric constant of PZT, AlN has still been pursued by many research groups at MEMS level $[7,8,9,10]$ due to the current nontrivial MEMS fabrication challenges of PZT MEMS $[11,12]$ and the presence of toxic lead [13] in PZT and other lead based piezoelectric materials.

In recent years, a few research groups [13, 14] have demonstrated that the lead-free KNN is a feasible candidate for MEMS piezoelectric VEH. Although the current experimental strain constant of KNN operates at same order of magnitude as AlN, its theoretical potential is predicated to be closer to that of PZT [15, 16]. Another group [17] also demonstrated the development of a continuous fabrication process for an entirely polymer-based piezoelectric film (PVDF based) at MEMS level.

Material selection aside, the popular topological design of choice for MEMS piezoelectric VEH todate remains that of a basic cantilever beam with an end mass [18]. This is mainly due to the simplicity of the design as well as the high quality factor achievable compared to the fundamental resonant mode of clamped-clamped alternatives. Although variations from the plain cantilever design do exist [19, 20, 21], all cantilever-based designs are primarily based on the principle of accumulating strain energy near the clamped end. One of the main parameters determining the effectiveness of a piezoelectric material as a generator is the strain constant $d$ as defined by equation 1 .

$$
d=\frac{\text { short circuit charge density }}{\text { applied mechanical stress }} ; \mathrm{C} \mathrm{N}^{-1}
$$


For a cantilever beam where the piezoelectric layer is sandwiched between two electrode layers, the $d_{31}$ mode applies; where induced polarisation is in the direction 3 (across the beam thickness) per unit induced stress in direction 1 (compression of tension along the long axis of the beam). Therefore, the objective is to maximise the induced stress or strain per unit area per unit force applied. Due to the decreasing bending strain distribution along the classical plain cantilever beam length, the addition of the proof mass near the free end can improve the compliance and thus the applied force, without significantly compromising the strain concentration regions near the clamped end.

While masses taking up varying proportions of the cantilever length exist in the literature $[22,23$, $24,4]$ and removing less strained areas of a cantilever beam from the active piezoelectric area has been shown to improve power output [25], a discussion of the optimal proof-mass-to-cantilever-length ratio has not been reported yet. For devices where the end mass is too small, the enhancement in power responsiveness stemmed from the addition of proof mass would be ineffective. However, if the mass occupies too much of the cantilever length, very small piezoelectric transduction area will remain active and significant regions of the otherwise high strain zones would be sacrificed. This paper numerically and experimentally explores the compromise between these two factors and proposes an optimal ratio between the cantilever length and the mass length.

\section{Design and modelling}

According to the classical beam theory, the elastic deflection amplitude $\delta_{x}$ of a cantilever beam in the transverse $x$ plane is given by Equation 2 .

$$
\delta_{x}=\frac{F l_{a}^{2}\left(3 l-l_{a}\right)}{6 E I}
$$

where, $F$ is the force applied to the free end tip of the beam, $l$ is the active length of the cantilever beam, $l_{a}$ is the length to point $a$ along $l$ from the clamped end, $E$ is the elastic modulus and $I$ is the area moment of inertia.

In the context of MEMS cantilevers, factors such as gravity is not influential while other factors that are considered negligible for macro-cantilevers such as internal stress becomes more dominant. Stoney's equation shown in Equation 3 can be used to approximate the deflection of MEMS cantilevers [26].

$$
\delta_{x}=\frac{3 \sigma l^{2}(1-\nu)}{E h^{2}}
$$

where, $\sigma$ is the applied stress, $\nu$ is the Poisson's ratio and $h$ is the thickness of the beam.
The natural frequency of cantilevers, both micro and macro, can be represented by Equation 4 [27].

$$
\omega_{0}=\sqrt{\frac{3 E I}{m l^{3}}}
$$

where, $m$ is the effective mass of the load at the free end of the beam. This $m$ directly feeds into $F$ in Equation 2 and $\sigma$ in Equation 3, along with acceleration and quality factor of the resonator. The fundamental mode natural frequency of an unloaded cantilever beam with significant beam mass $m_{b}$ is given by Equation 5 [27].

$$
\omega_{0}=1.875^{2} \sqrt{\frac{E I}{m_{b} l^{3}}}
$$

Therefore, by substituting Equation 5 back into Equation 4, the effective mass of the beam alone is given by $0.2427 m_{b}$. Henceforth, the natural frequency of a cantilever beam with significant beam mass $m_{b}$ as well as load mass $m_{l}$ is given by Equation 6 .

$$
\omega_{0}=\sqrt{\frac{3 E I}{\left(0.2427 m_{b}+m_{l}\right) l^{3}}}
$$

For a plain cantilever beam without a proof mass, the typical strain distribution along the cantilever beam length when subjected to a loading is illustrated in figure 1. Origin denotes the clamped end.

By computing the average induced strain across the piezoelectric layer, the charge generation can be calculated by equation 7 and peak power can be estimated by equation 8 .

$$
\begin{gathered}
q=d_{31} \epsilon_{p a v} E w_{p} l_{p} \\
P=\frac{\omega h_{p} q^{2}}{\epsilon_{0} \epsilon_{r} w_{p} l_{p}}
\end{gathered}
$$

where, $q$ is the electric charge generated, $d_{31}$ is the piezoelectric strain constant in the 31 mode, epav is the average strain experienced in the piezoelectric layer, $w_{p}, l_{p}$ and $h_{p}$ are the width, length and thickness of the piezoelectric layer, $E$ is the elastic modulus, $P$ is the power aptitude achievable at frequency $\omega, \epsilon_{0}$ is the permittivity of air and $\epsilon_{r}$ is the dielectric constant of the piezoelectric material.

It can be seen from figure 1 and equation 7 that the differential strain distributions contribute unequally to the charge generated. In fact, the inclusion of the area near the free end as part of the active piezoelectric transducer dilutes the average strain value in equation 7. Additionally, the increase in capacitive area negatively contributes to the power output in equation 2 . Only the $\sim 1 / 3 \mathrm{rd}$ of the cantilever beam near the clamped end contributes positively to the accumulation of average 

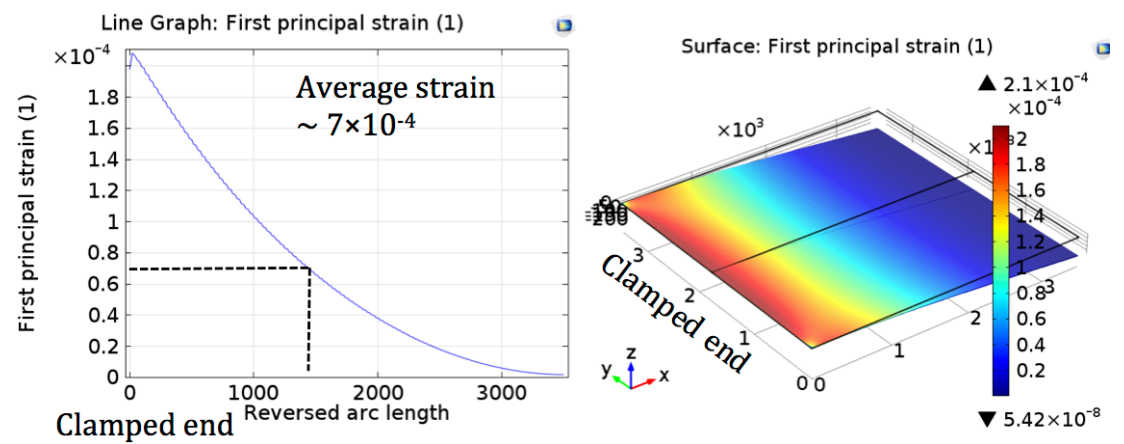

Figure 1: Typical strain distribution of a plain cantilever. The stationary response is computed for a load of $1.5 \mathrm{mN}$. Only approximately the first third of the cantilever beam near the clamped end contribute positively to the average strain.

strain as shown by the dotted lines in figure 1. Therefore, based on this rationale, a significant portion of the free end can be sacrificed to house a stiff mass without compromising the effective transduction area.

Four cantilevers with varying proof mass sizes were designed, modelled, fabricated and experimentally characterised to establish the optimal relationship between cantilever length and proof mass length. Figure 2 shows the four cantilevers with $2 \mathrm{D}$ dimensions of $3.5 \mathrm{~mm}$ by $3.5 \mathrm{~mm}$ for each device. The un-etched silicon substrate $(400 \mu \mathrm{m})$ was used as the proof mass to create the 4 variants: MC40, MC50, MC60 and MC70 (number denoting percentage of the cantilever device length occupied by the end mass).

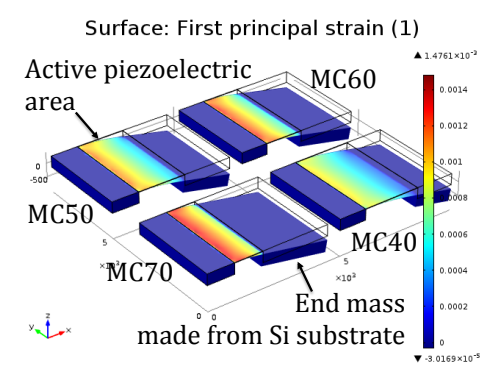

Figure 2: COMSOL FEA model of the MEMS microcantilevers with varying end mass. The number denotes percentage of the cantilever length taken up by the mass.

Analytical and numerical models were constructed using the classical theory equations for resonant cantilever harvesters (outlined in figure 3 ) in order to compute the resultant strain for various design iterations. Equation 9 is used to numerically (in MATLAB) analyse the response of the resonator for a given quality factor. Additionally, strain values calculated from beam equations and extracted from FEA models were used to both analytically and numerically evaluate the power response.

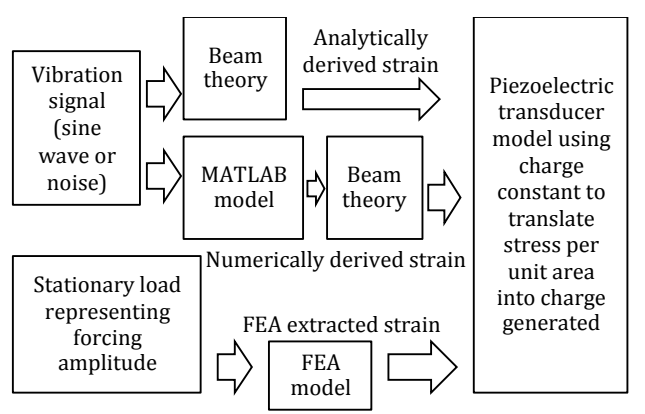

Figure 3: Outline of analytical, numerical and FEA models used to analysis power output of the various cantilever prototypes. Note, noise signal was only fed into the MATLAB model.

$$
\ddot{x}+2 \zeta_{1} \omega_{n} \dot{x}+\zeta_{2} \dot{x}|\dot{x}|+\mu x^{3}+\omega_{n}^{2} x=A \omega^{2} Q \cos \omega t
$$

where, $x$ is the displacement, $\zeta_{1}$ is the viscous damping, $\zeta_{2}$ is the quadratic damping representing nonlinear air drag, $\mu$ is the Duffing coefficient, $\omega_{n}$ is the natural frequency, $A$ is the excitation displacement amplitude, $\omega$ is the excitation frequency, $Q$ is the quality factor of the resonator and $t$ is the time domain.

Analytically calculated results for the proofmass-to-cantilever-length ratio is presented in figures 4 and 5 . The highest power response per unit acceleration was found for designs where the end mass takes up around $65 \%$ of the cantilever length; and this relationship holds for varying cantilever dimensions as shown in the plot. A fast diminishing return is predicted for end mass taking up higher than $\sim 80 \%$ of the total beam length, as significant regions of the effective strain areas are sacrificed for the proof mass. An optimal configuration is suggested for cantilevers where approximately $60 \%$ to $70 \%$ of the beam length is occupied by the proof mass.

Figure 6 illustrates the FEA strain distribution (for the devices shown in figure 2) along the 3.5 


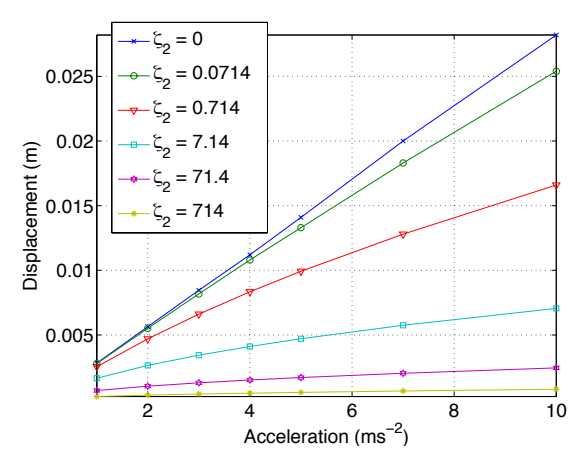

Figure 4: Analytically calculated voltage response per $1 \mathrm{~g}$ of acceleration, to determine the ideal proof mass to cantilever length ratio, for an assumed Q factor of 40 .

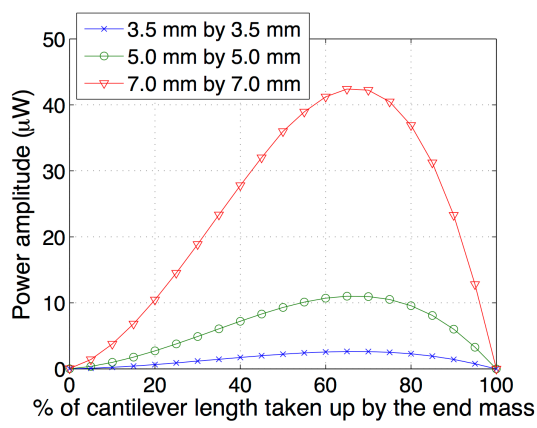

Figure 5: Analytically calculated power response per $1 \mathrm{~g}$ of acceleration, to determine the ideal proof mass to cantilever length ratio, for an assumed Q factor of 40 .

$\mathrm{mm}$ cantilever total length. As expected, strain is nullified for the area where end mass is present. Therefore, while peak strain increases for larger masses, the resultant strain spans less area. For the same amount of loading, it can be seen that MC70 and MC60 experiences higher average strain than MC40 and MC30 despite the smaller effective bending area.

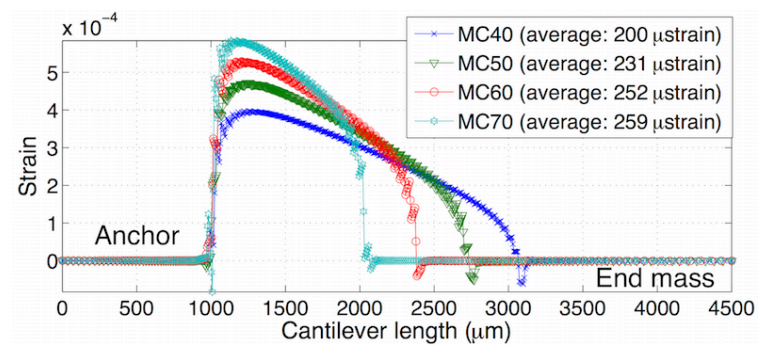

Figure 6: FEA simulated strain response for the four $3.5 \mathrm{~mm}$ long micro-cantilevers with varying end mass, when subjected to the same loading.

\section{Method and result}

\section{Apparatus}

The micro-cantilevers were fabricated using a 0.5 $\mu \mathrm{m}$ AlN on $10 \mu \mathrm{m}$ doped Si process as outlined in figure 7. Due to the potential for large displacement from the micro-cantilevers, the MEMS chip was assembled on top of an aluminium spacer $(\sim 1.3 \mathrm{~mm}$ tall) within a deep cavity chip carrier (3 $\mathrm{mm}$ deep). This accommodated unrestricted travel of about \pm 1 $\mathrm{mm}$ and no physical limit saturation was recorded for the scanned acceleration range reported in this paper.

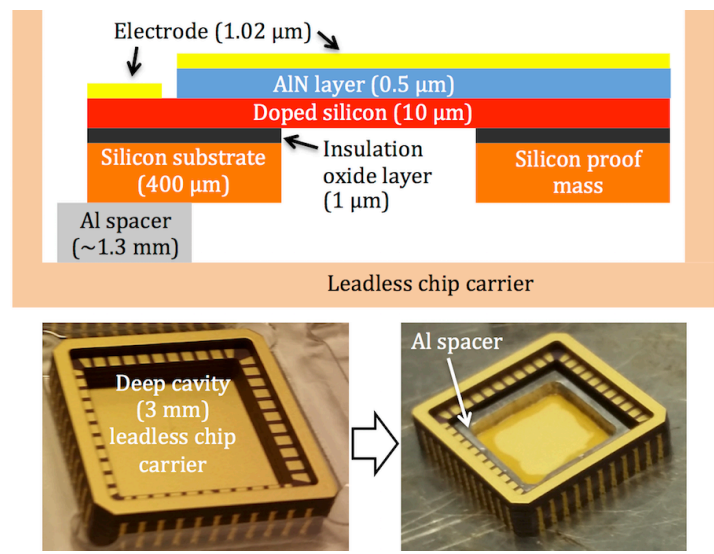

Figure 7: Device and package cross-sectional view. A spacer is used to accommodate large travel.

Figure 8 presents the photograph of the 4 microcantilever prototypes under test on a vibration shaker. Despite sharing the same chip, the 4 devices are mechanically and electrically uncoupled, and measurements were taken separately. Table 1 delineates the calculated (beam equations), simulated (COMSOL FEA) and measured natural frequency values for the 4 devices.

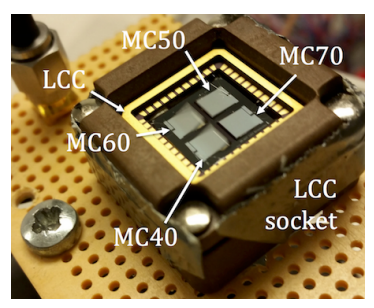

Figure 8: Photograph of the MEMS chip consisting of the 4 uncoupled micro-cantilevers with varying end mass.

The experimental frequency measurements were undertaken by a laser Doppler vibrometer where the cantilevers were optically measured while electrically driven by a periodic chirp, as well as electrically measured on an oscilloscope when mechanically driven by a shaker that is controlled by a function generator. 

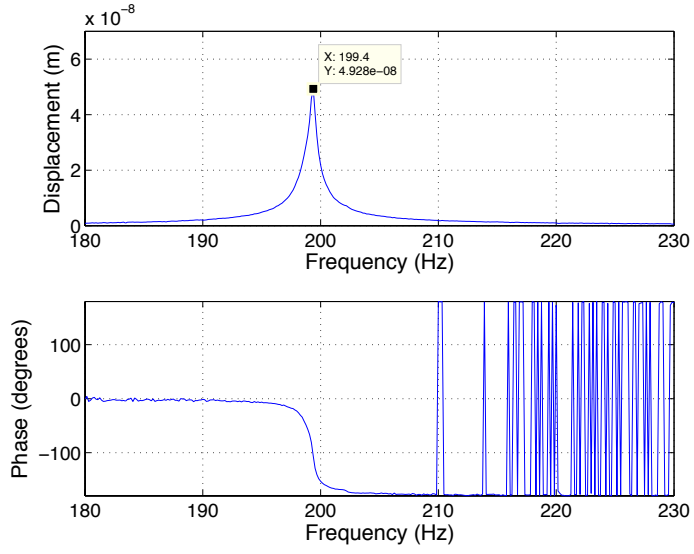

(a) MC40, peaks at $199.4 \mathrm{~Hz}$ and $49 \mathrm{~nm}$
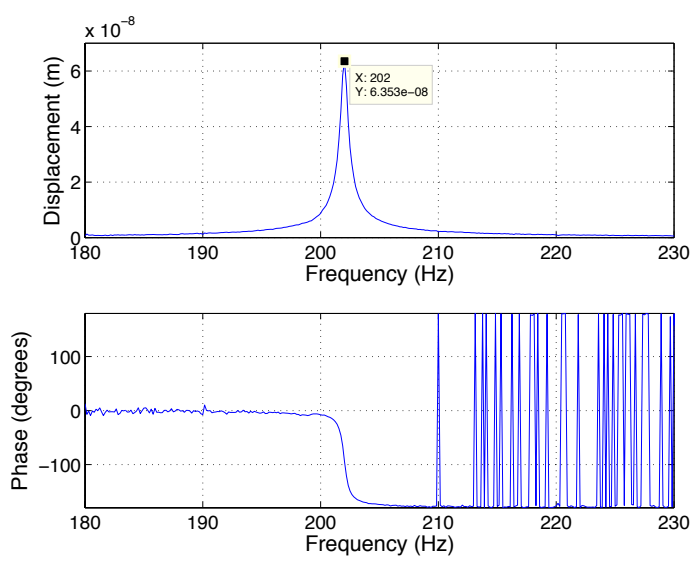

(c) MC60, peaks at $202.0 \mathrm{~Hz}$ and $64 \mathrm{~nm}$
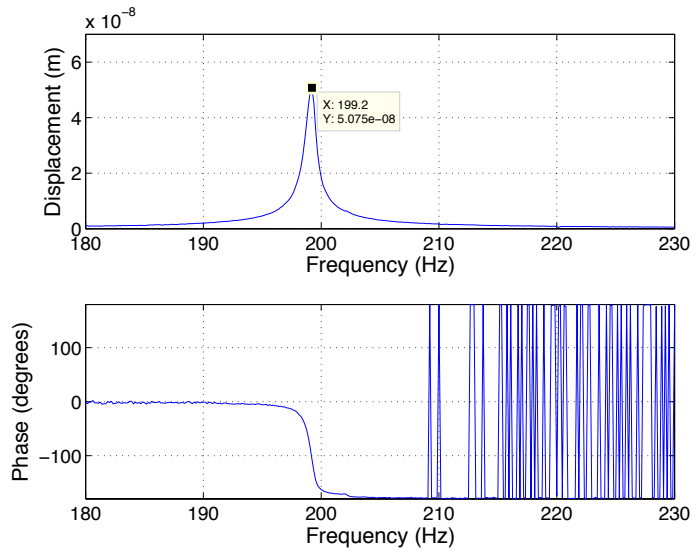

(b) MC50, peaks at $199.2 \mathrm{~Hz}$ and $51 \mathrm{~nm}$
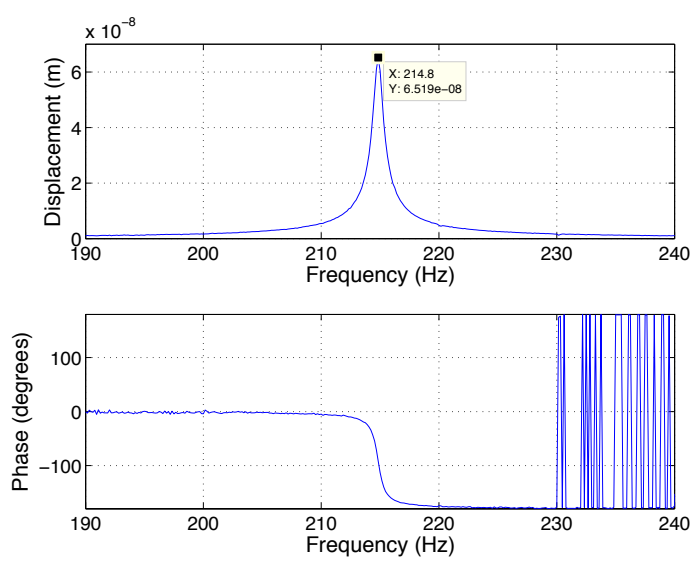

(d) MC70, peaks at $214.8 \mathrm{~Hz}$ and $65 \mathrm{~nm}$

Figure 9: Frequency domain response of the four micro-cantilevers with varying end mass measured by laser Doppler vibrometer. All measurements were taken for a periodic chirp of $0.5 \mathrm{~V}_{a c}$. Floor-level noise, measured from an anchored point, has been subtracted from all the plots.

Table 1: Natural frequencies according to beam equations, COMSOL solid mechanics simulation (FEA), and measurements from laser Doppler vibrometer (LDV) and shaker.

\begin{tabular}{c|cccc}
\multirow{2}{*}{ Device } & \multicolumn{4}{|c}{ Natural frequency $(\mathrm{Hz})$} \\
& Eqn. & FEA & LDV & shaker \\
\hline MC40 & 213.65 & 223.4 & 199.38 & 199.9 \\
MC50 & 212.61 & 220.2 & 199.22 & 199.2 \\
MC60 & 216.87 & 224.6 & 202.03 & 202.2 \\
MC70 & 225.81 & 238.3 & 214.84 & 215.0
\end{tabular}

\section{Linear response}

The frequency domain response of the 4 devices, measured by laser Doppler vibrometer, is presented in figure 9. A periodic chirp of $0.5 \mathrm{~V}_{a c}$ spanning between $50 \mathrm{~Hz}$ and $1 \mathrm{kHz}$ was used as the electrical drive input signal. The mechanical response was optically measured from the tips of the 4 microcantilevers. It can be seen that given the same excitation, the yielded resonant response ranks in an ascending order of MC40, MC50, MC60 and MC70 respectively. Moreover, MC60 and MC70 can be seen to notably more responsive than MC40 and MC50.

Figure 10 presents a snapshot of the experimentally measured power response, from the vibration shaker, in the frequency domain for the four prototypes across matched resistive loads (around 0.3 $\mathrm{M} \Omega$ ) at a fixed $3.4 \mathrm{~ms}^{-2}$ drive acceleration. The Duffing nonlinearity was observed to be more significant for cantilevers with smaller masses, as MC50 and MC40 had relatively more noticeable bending of the resonant peaks. The responsiveness ranking of the mechanical-to-electrical case is thus far consistent with the electrical-to-mechanical response.

Figure 11a shows the power per input acceleration response at the respective natural frequencies of the micro-cantilevers. Agreement with the analytical and FEA models is shown within the linear region at low acceleration levels, where MC70 and MC60 outperforms MC50 and MC40. 


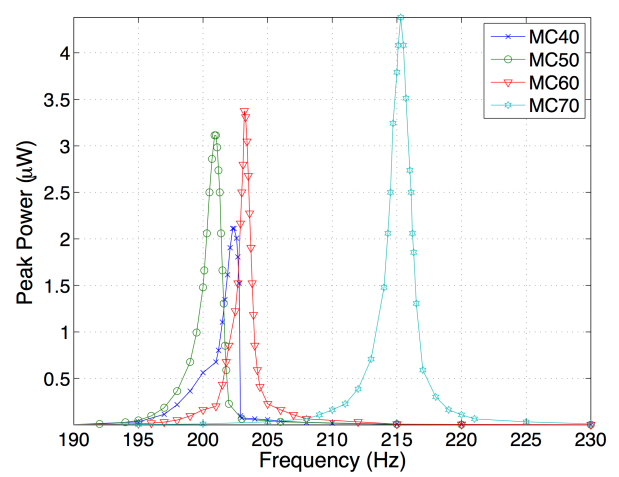

Figure 10: Measured frequency domain power response for the four cantilevers at $3.4 \mathrm{~ms}^{-2}$ of sinusoidal drive.

MC70 delivered $1.18 \mu \mathrm{W}(0.59 \mathrm{~V})$ of peak power when subject to acceleration amplitude of 1.23 $\mathrm{ms}^{-2}$ at $215 \mathrm{~Hz}$. At comparable levels of acceleration $\left(1.72 \mathrm{~ms}^{-2}\right)$, MC60 recorded $1.14 \mu \mathrm{W}(0.57$ $\mathrm{V})$ of peak power at $202 \mathrm{~Hz}$. On the other hand, MC50 was able to provide $0.85 \mu \mathrm{W}(0.50)$ at 1.72 $\mathrm{ms}^{-2}$ and MC40 was able to supply $0.48 \mu \mathrm{W}(0.38$ $\mathrm{V})$ at $1.55 \mathrm{~ms}^{-2}$. For a practical device volume of about $5 \mathrm{~mm}^{3}$, table 2 compares the power density per acceleration squared for the four prototypes.

\section{Nonlinear damping}

Although MC70 is seen to have a significantly higher power density per acceleration squared, at large acceleration levels (figure 11b), diminishing returns were observed for the larger mass device as the power curves start to flatten off. Since the devices were all tested in atomspheric pressure, nonlinear damping would onset at large deflections as the film-like cantilever resonators squeeze the air trapped underneath it within the bottom-sealed deep cavity chip carrier.

The squeeze film damping effect adversely affects larger mass resonators more significantly, such as MC70 and MC60, as these devices are able to attain larger deflection for smaller input acceleration levels and have larger surface area (mass blocks) [28].

Figure $11 \mathrm{~b}$ shows that MC70 is the first to flatten off after approximately $3.5 \mathrm{~ms}^{-2}$ of acceleration, while MC60 also exhibited a similar behaviour at around $5.5 \mathrm{~ms}^{-2}$. This is further evidenced by the frequency domain snapshot for approximately 0.5 $\mathrm{g}$ of input acceleration shown in figure 12. Therefore, within the nonlinear regions, the larger mass devices starts to lose its advantage over MC40 and MC50. In fact, all of the other three cantilevers outperformed MC70 beyond $\sim 4 \mathrm{~ms}^{-2}$.

To better understand this behaviour, a numerical simulation based on equation 9 was undertaken for

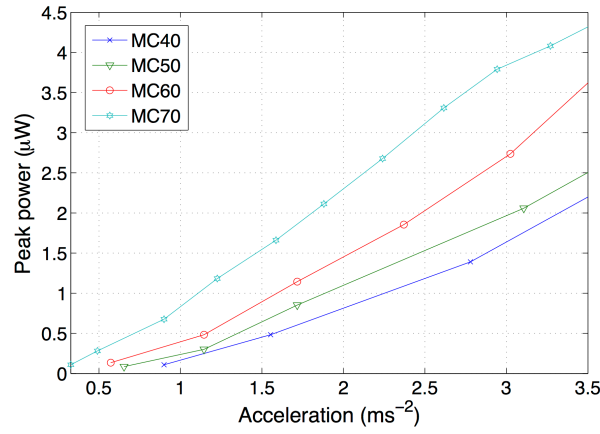

(a) Within linear region

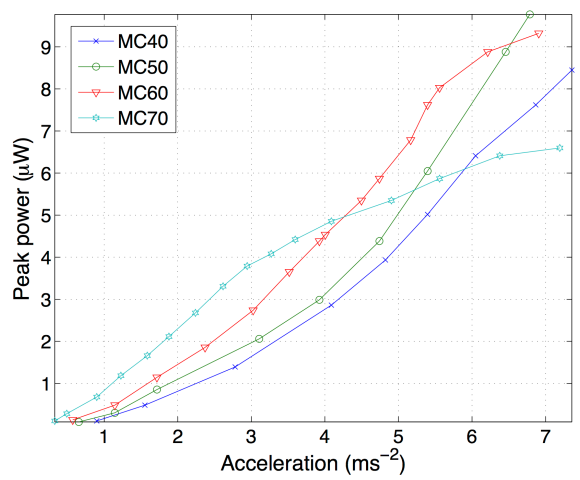

(b) Full range

Figure 11: Measured peak power response as a function of input acceleration at the natural frequency.

varying nonlinear quadratic damping (figure 13). The simulation shows a linear response for zero nonlinear damping. However, with the introduction of nonlinear damping, the curve starts to flatten off at larger amplitudes. This effect is clearly illustrated by a weakly nonlinearly damped scenario, where the response is approximately linear at small excitations but starts to experience the diminishing returns at higher amplitudes.

\section{High power configuration}

In order to validate the hypothesis that the source of the observed nonlinear damping is indeed the film-squeezing action of the trapped air within the package, a MEMS chip was assembled on a bottomhollowed leadless chip carrier (figure 14) and tested on a shaker. Despite not being vacuum-packaged, this setup allowed free flowing of air and nullified the effects of air drag from potential film-squeezing actions.

Figure 15 compares the power response of the four micro-cantilevers at both bottom-sealed and bottom-holed assembly scenarios. It is seen that the sample devices significantly outperformed their corresponding twins when assembled on the bottom-holed setup. Additionally, the flattening off the power curves, indicating the onset of non- 
Table 2: Power density normalised against acceleration squared for the four micro-cantilevers within linear operational region when assembled in a deep cavity bottom-sealed chip carrier. All devices have a volume of around $5 \mathrm{~mm}^{3}$

\begin{tabular}{c|ccccc} 
Device & $\begin{array}{c}\text { Voltage } \\
(\mathrm{V})\end{array}$ & $\begin{array}{c}\text { Power } \\
(\mu \mathrm{W})\end{array}$ & $\begin{array}{c}\text { Acceleration } \\
\left(\mathrm{ms}^{-2}\right)\end{array}$ & $\begin{array}{c}\text { Frequency } \\
(\mathrm{Hz})\end{array}$ & $\begin{array}{c}\mathrm{NPD} \\
\left(\mu \mathrm{Wcm}^{-3} \mathrm{~m}^{-2} \mathrm{~s}^{4}\right)\end{array}$ \\
\hline MC70 & 0.59 & 1.18 & 1.23 & 215.0 & 157 \\
MC60 & 0.57 & 1.14 & 1.72 & 202.2 & 77 \\
MC50 & 0.50 & 0.85 & 1.72 & 199.2 & 58 \\
MC40 & 0.38 & 0.48 & 1.55 & 199.9 & 40
\end{tabular}

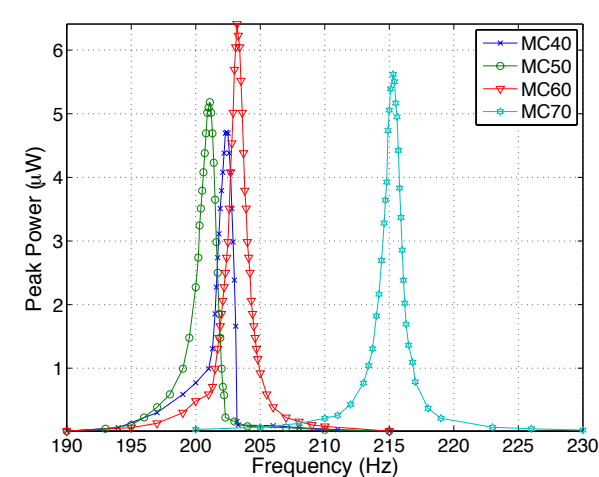

Figure 12: Measured frequency domain power response for the four cantilevers at $\sim 5 \mathrm{~ms}^{-2}$ of sinusoidal drive.

linear damping, was not noted for the bottom-holed configuration.

All devices in the holed LCC setup soon fractured after scanning beyond the presented acceleration range. A few selected power response at a low and relatively higher acceleration inputs are presented in table 3. With minimised nonlinear damping effects, the power responsiveness and the normalised power density ranking remained constant throughout the amplitude range. Thus, this establishes the superiority of MC70 and MC60 over MC50 and MC40 in terms of peak power performance.

Additionally, as can be seen from table 3, the normalised power density of the prototypes tested in the bottom-holed chip carrier (free airflow) outperformed its bottom-sealed (nonlinear damped) counterparts by nearly an order of magnitude. MC70 assembled on bottom-holed chip carrier was able to produce $1.78 \mu \mathrm{W}$ at $0.60 \mathrm{~ms}^{-2}$ $\left(989 \mu \mathrm{Wcm}^{-3} \mathrm{~m}^{-2} \mathrm{~s}^{4}\right)$, while an identical prototype mounted on a bottom-sealed chip carrier only generated $1.18 \mu \mathrm{W}$ at about twice the acceleration level $\left(1.23 \mathrm{~ms}^{-2}\right.$ and $\left.157 \mu \mathrm{Wcm}^{-3} \mathrm{~m}^{-2} \mathrm{~s}^{4}\right)$. Moreover, MC70 produced up to $20.47 \mu \mathrm{W}$ at $2.7 \mathrm{~ms}^{-2}$, while the device saturated in the vicinity of $6 \mu \mathrm{W}$ to 7 $\mu \mathrm{W}$ after around $6 \mathrm{~ms}^{-2}$.

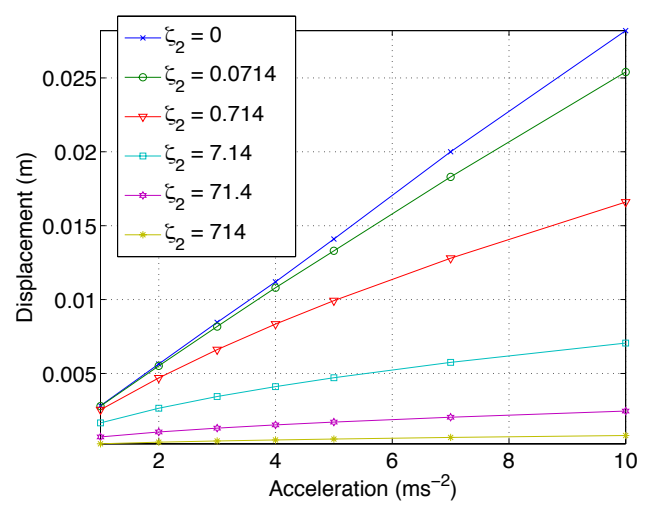

(a) Flattening of response with increasing $\zeta_{2}$

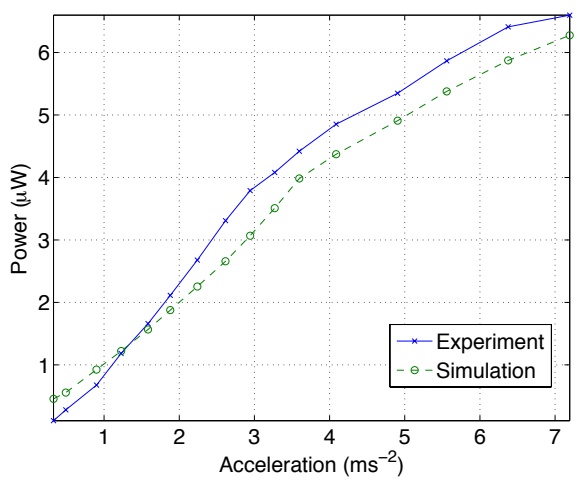

(b) MC70 power output fit

Figure 13: Numerically simulated response of a microcantilever when subjected to varying nonlinear damping. Fixed parameter values: $f_{0}=210 \mathrm{~Hz}, \zeta_{1}=0.0071$ $\mathrm{Nsm}^{-1}$ and $\mu=-0.1 \mathrm{Nm}^{-3}$; and $\zeta_{2}=71.4 \mathrm{Nsm}^{-1}$ for the MC70 power output fit.

\section{Noise response}

Apart from the resonant response, figure 17 further compares the average power output amongst the 4 micro-cantilever prototypes were subjected to band-limited white noise vibration. The result once again agrees with the models in terms of the relative power per acceleration superiority of MC70 and MC60 over MC50 and MC40. No mass-dependent diminishing returns from nonlinear damping was observed for the white noise driven cases, as a result of the relatively smaller associated cantilever displacements. 
Table 3: Power density normalised against acceleration squared for the four micro-cantilevers when assembled in a bottom-holed chip carrier (free airflow). All devices have a volume of around $5 \mathrm{~mm}^{3}$

\begin{tabular}{c|ccccc} 
Device & $\begin{array}{c}\text { Voltage } \\
(\mathrm{V})\end{array}$ & $\begin{array}{c}\text { Power } \\
(\mu \mathrm{W})\end{array}$ & $\begin{array}{c}\text { Acceleration } \\
\left(\mathrm{ms}^{-2}\right)\end{array}$ & $\begin{array}{c}\text { Frequency } \\
(\mathrm{Hz})\end{array}$ & $\begin{array}{c}\mathrm{NPD} \\
\left(\mu \mathrm{Wcm}^{-3} \mathrm{~m}^{-2} \mathrm{~s}^{4}\right)\end{array}$ \\
\hline \multirow{2}{*}{ MC70 } & 0.73 & 1.78 & 0.60 & 210 & 989 \\
& 2.48 & 20.47 & 2.7 & & 562 \\
\hline \multirow{2}{*}{ MC60 } & 0.75 & 1.88 & 0.65 & 196 & 890 \\
& 2.45 & 20.06 & 2.7 & & 550 \\
\hline \multirow{2}{*}{ MC50 } & 0.83 & 2.27 & 0.90 & \multirow{2}{*}{193} & 560 \\
& 2.43 & 19.74 & 2.9 & & 469 \\
\hline \multirow{2}{*}{ MC40 } & 0.50 & 0.84 & 0.65 & \multirow{2}{*}{199} & 398 \\
& 2.31 & 17.83 & 3.0 & & 396
\end{tabular}

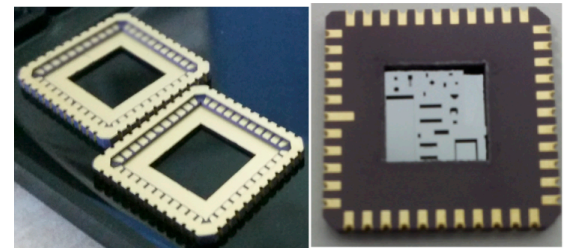

Figure 14: Laser micro-machined bottom-hollowed leadless chip carrier (LCC) to minimise nonlinear squeeze film damping of air within the bottom-sealed LCC. (Note, the image is for illustration only using a dummy chip).

Figure 17 illustrates the time domain response of the micro-cantilevers when subjected to $7 \mathrm{~g}$ of band limited white noise $\left(0.025 \mathrm{~g}^{2} \mathrm{~Hz}^{-1}\right)$. The average power response were $6.3 \mathrm{nW}, 8.2 \mathrm{nW}, 11.3$ $\mathrm{nW}$ and 14.3 for MC40, MC50, MC60 and MC70 respectively.

\section{Discussion}

\section{Package}

It has been demonstrated that the adoption of larger cantilever masses can yield better power responsiveness, especially towards a configuration that occupies around $60 \%$ to $70 \%$ of the cantilever beam length. However, the larger mass within a closed package with non-trivial air pressure result in the onset of the nonlinear squeezing film damping.

While this effect reduces the absolute power per unit acceleration, it can also serve as a 'soft' mechanical stopper to limit the amplitude of the resonator. This in turn helps the mechanical harvesters to survive at high acceleration levels. Amongst the cantilever prototypes surveyed, it can be seen that while the hollow LCC package allowed the cantilever harvesters to attain higher power output at relatively small acceleration input, the cantilevers also readily fractured at small excitation levels. On the other hand, the power output of the cantilever harvesters plateaued after a certain

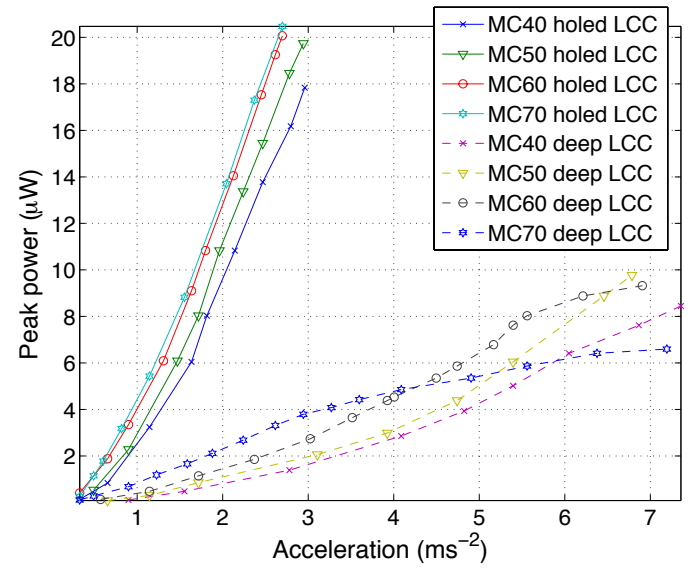

Figure 15: Measured peak power response for the four micro-cantilvers when assembled on a bottom-sealed deep cavity LCC (presence of nonlinear air damping) and a bottom-hollowed LCC (free airflow)

acceleration level, but the devices remained intact.

\section{Comparison with the literature}

The results of the 4 micro-cantilever variants within both the deep cavity package and the holed chip carrier are compared to other piezoelectric cantilever-based vibration energy harvesters reported in the literature (table 4). Two metrics popularly used in the literature are used for comparison: normalised power density (NPD) [1] and Figure of Merit (FOM) [4]. While these two metrics have been used in the literature, a standardised metric has yet to be established in the VEH field.

The proportion of the end mass to the beam length for each literature device was estimated from either text or figures. It can be seen that the linear region response of MC70 within the deep cavity chip carrier compares favourably to one of the best case devices in the literature [4], which employs thinned bulk PZT on silicon and also has approximately $70 \%$ of the beam devoted to the end mass.

Additionally, all of the devices mounted on the holed LCC noticeably outperformed both the deep 
Table 4: Comparison of the harvester prototypes with the literature. NPD is power density normalised against acceleration squared, $l_{m}$ denotes percentage of cantilever length taken up by end mass, $P$ is the peak power measured at frequency $f$ and drive amplitude Acc. FOM is figure of merit by multiplying NPD with the half power bandwidth $(-3 \mathrm{~dB})$. For literature data points where $-3 \mathrm{~dB}$ value was not given, an estimate was made based on the presented data.

\begin{tabular}{c|cccccccc} 
VEH & $\begin{array}{c}l_{m} \\
(\%)\end{array}$ & $\begin{array}{c}P \\
(\mu \mathrm{W})\end{array}$ & $\begin{array}{c}\text { Acc. } \\
\left(\mathrm{ms}^{-2}\right)\end{array}$ & $\begin{array}{c}V o l . \\
\left(\mathrm{cm}^{3}\right)\end{array}$ & $\begin{array}{c}f \\
(\mathrm{~Hz})\end{array}$ & $\begin{array}{c}-3 \mathrm{~dB} \\
(\mathrm{~Hz})\end{array}$ & $\begin{array}{c}\text { NPD } \\
\left(\mu \mathrm{W} \cdot \mathrm{cm}^{-3} \mathrm{~m}^{-2} \mathrm{~s}^{4}\right)\end{array}$ & $\begin{array}{c}\text { FOM } \\
(\mathrm{NPD} \cdot \mathrm{Hz})\end{array}$ \\
\hline MC70 holed LCC & $\sim 70$ & 1.78 & 0.60 & $5.0 \mathrm{E}-3$ & 210 & 1.0 & $9.9 \mathrm{E} 2$ & $9.89 \mathrm{E} 2$ \\
MC60 holed LCC & $\sim 60$ & 1.88 & 0.65 & $5.0 \mathrm{E}-3$ & 196 & 1.1 & $8.9 \mathrm{E} 2$ & $9.79 \mathrm{E} 2$ \\
MC50 holed LCC & $\sim 50$ & 2.27 & 0.90 & $5.0 \mathrm{E}-3$ & 193 & 1.2 & $5.6 \mathrm{E} 2$ & $6.73 \mathrm{E} 2$ \\
MC40 holed LCC & $\sim 40$ & 0.84 & 0.65 & $5.0 \mathrm{E}-3$ & 199 & 1.2 & $4.0 \mathrm{E} 2$ & $5.98 \mathrm{E} 2$ \\
MC70 deep LCC & $\sim 70$ & 1.18 & 1.23 & $5.0 \mathrm{E}-3$ & 215 & 2.0 & $1.6 \mathrm{E} 2$ & $3.12 \mathrm{E} 2$ \\
Aktakka (2011) [4] & $\sim 70$ & 2.74 & 0.98 & $2.7 \mathrm{E}-2$ & 167 & 6.1 & $1.1 \mathrm{E} 2$ & $6.45 \mathrm{E} 2$ \\
MC60 deep LCC & $\sim 60$ & 1.14 & 1.72 & $5.0 \mathrm{E}-3$ & 202 & 2.2 & $7.7 \mathrm{E} 1$ & $1.70 \mathrm{E} 2$ \\
Erturk (2008) [29] & $\sim 20$ & $2.4 \mathrm{E} 4$ & 9.81 & 3.52 & 45.6 & 2.5 & $7.1 \mathrm{E} 1$ & $1.77 \mathrm{E} 2$ \\
Roundy (2003) [30] & $\sim 60$ & 375 & 2.5 & 1.00 & 120 & 3.6 & $6.0 \mathrm{E} 1$ & $2.16 \mathrm{E} 2$ \\
MC50 deep LCC & $\sim 50$ & 0.85 & 1.72 & $5.0 \mathrm{E}-3$ & 199 & 2.4 & $5.8 \mathrm{E} 1$ & $1.38 \mathrm{E} 2$ \\
MC40 deep LCC & $\sim 40$ & 1.39 & 2.78 & $5.0 \mathrm{E}-3$ & 200 & 2.6 & $3.6 \mathrm{E} 1$ & $9.35 \mathrm{E} 1$ \\
Besse (2012) [3] & $\sim 50$ & 63.7 & 9.81 & $1.9 \mathrm{E}-2$ & 41.1 & 2.2 & $3.5 \mathrm{E} 1$ & $7.66 \mathrm{E} 1$ \\
Zhu (2011) [31] & $\sim 40$ & 240 & 3.9 & $5.5 \mathrm{E}-1$ & 67 & 2.0 & $2.9 \mathrm{E} 1$ & $5.74 \mathrm{E} 1$ \\
Elfrink (2009) [7] & $\sim 80$ & 60 & 19.6 & $1.2 \mathrm{E}-2$ & 572 & 3.5 & $1.3 \mathrm{E} 1$ & $4.56 \mathrm{E} 1$ \\
Microgen (2013) [32] & $\sim 60$ & 900 & 9.8 & $7.2 \mathrm{E}-1$ & 600 & 2.0 & $1.3 \mathrm{E} 1$ & $2.60 \mathrm{E} 1$ \\
Andosca (2012) [23] & $\sim 60$ & 128 & 9.8 & $2.6 \mathrm{E}-1$ & 58 & 2.0 & $5.2 \mathrm{E} 0$ & $1.03 \mathrm{E} 1$
\end{tabular}

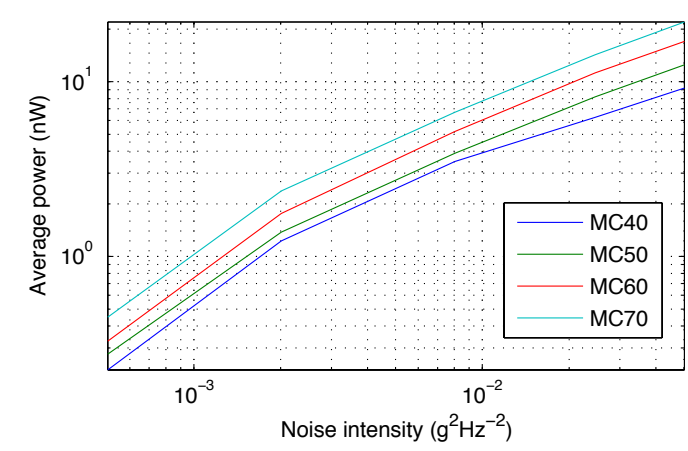

Figure 16: Measured average power response for the 4 micro-cantilever prototypes when subjected to bandlimited white noise vibration input $(10 \mathrm{~Hz}$ to $2 \mathrm{kHz})$.

LCC devices and the literature devices. The normalised power density of MC70 and MC60 tested in the holed-LCC (free airflow) recorded up to nearly an order of magnitude higher than the thinned bulk PZT device [4].

Solely comparing the literature devices, with the exception of [29] (which compromised of bimorph) and [32, 23] (from the same group), the general trend shows favour towards cantilevers with $\sim 70 \%$ and $\sim 60 \%$ of the length taken up by the end mass compared to those with $\sim 40 \%, \sim 50 \%$ and $\sim 80 \%$ of end mass. This indirectly also supports the findings concluded in this paper.

\section{Conclusion and future work}

This paper presents design insight into power optimisation for piezoelectric cantilever vibration energy harvesters based on experimentally validated analytical and numerical analysis. It is observed that the ideal proportion of the end mass should occupy approximately $60 \%$ to $70 \%$ of the beam length. However, vacuum packaging or bottomhollowed packages are required to minimise nonlinear air damping at large deflections, which adversely affects the larger massed devices more significantly.

A peak power output of $1.78 \mu \mathrm{W}$ at $0.6 \mathrm{~ms}^{-2}$ and up to $20.5 \mu \mathrm{W}$ at $2.7 \mathrm{~ms}^{-2}(210 \mathrm{~Hz})$ was recorded for an AlN-on-SOI cantilever with $70 \%$ of its beam dedicated to housing a silicon end mass. This result outperformed one of the previously reported best case devices from the literature by nearly an order of magnitude in terms of power density per acceleration squared $\left(989 \mu \mathrm{Wcm}^{-3} \mathrm{~m}^{-2} \mathrm{~s}^{4}\right.$ compared to $\left.105 \mu \mathrm{Wcm}^{-3} \mathrm{~m}^{-2} \mathrm{~S}^{4}[4]\right)$.

Future work will investigate the power performance of the packaged device under vacuum conditions, in an attempt to further enhance the power responsiveness of the proof mass optimised piezoelectric vibration energy harvesters. Additionally, interface circuitry will be investigated to ensure optimal conversion efficiency of the harvested raw power into useful regulated power. 

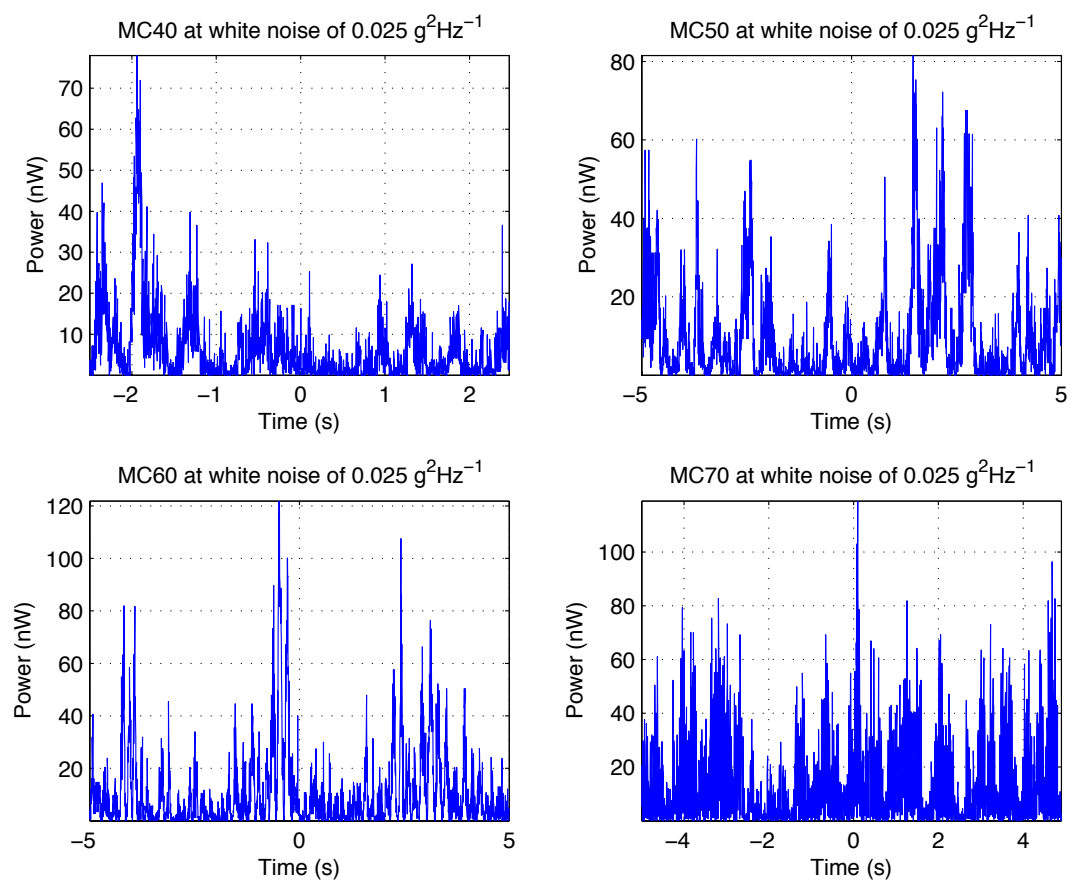

Figure 17: Time domain power response for the 4 micro-cantilever prototypes when subjected to $0.025 \mathrm{~g}^{2} \mathrm{~Hz}^{-1}$.

\section{Acknowledgement}

This work was supported by Innovate UK and GE Aviation.

\section{References}

[1] S. Beeby, M. Tudor, and N. White, "Energy harvesting vibration sources for microsystems applications," Meas. Sci. Technol., vol. 17, no. 12, pp. R175-R195, 2006.

[2] S. Priya and D. Inman, Energy Harvsting Technologies. New York: Springer US, 2009.

[3] N. Besse, A. Quintero, D. Briand, P. Janphuang, R. Lockhart, J. Ruan, and N. de Rooij, "Pztbased energy harvesters on plastic foil optimized through theoretical modeling and fabrication improvements," in PowerMEMS, Atlanta, Georgia, USA, Dec. 02 - 05 2012, pp. pp. 42-45.

[4] E. Aktakka, R. Peterson, and K. Najafi, "Thinnedpzt on soi process and design optimization for piezoelectric inertial energy harvesting," in Transducers, Beijing, China, June 5-9 2011, pp. pp. 1649-1652.

[5] S. Kok, N. White, and N. Harris, "Fabrication and characterization of free-standing thick-film piezoelectric cantilevers for energy harvesting," Meas. Sci. Technol., vol. 20, no. 12, p. 13pp., 2009.

[6] A. Khaligh, P. Zeng, and C. Zheng, "Kinetic energy harvesting using piezoelectric and electromagnetic technologies - state of the art," IEEE Trans. Ind. Electron., vol. 57, no. 3, pp. pp. $850-860$, 2010.
[7] R. Elfrink, T. M. Kamel, M. Goedbloed, S. Matova, D. Hohlfeld, Y. van Andel, and R. van Schaijk, "Vibration energy harvesting with aluminum nitride-based piezoelectric devices," J. Micromech. Microeng, vol. 19, no. 9, p. 8pp., 2009.

[8] Q. Wang, Z. Cao, and H. Kuwano, "Vibration energy harvesters based on c-axis tilted aln thin films," in PowerMEMS, Atlanta, Georgia, USA, Dec. 02 - 05 2012, pp. pp. 327-330.

[9] Z. Wang, R. Elfrink, M. Renaud, R. Vullers, and R. van Schaijk, "Study of piezoelectric vibration harvester based on clamped-clamped beams under shock excitation," in PowerMEMS, Atlanta, Georgia, USA, Dec. 02 - 05 2012, pp. pp. 46-49.

[10] Y. Lai, W. Li, C. Lin, V. Felmetsger, and A. Pisano, "High-temperature stable piezoelectric aluminum nitride energy harvesters utlizing elastically supported diaphragms," in Transducers, Barcelona, Spain, June 16-20 2013, pp. pp. 22682271.

[11] H. Bardaweel, O. A. Hattamleh, R. Richards, D. Bahr, and C. Richards, "A comparison of piezoelectric material for mems power generation," in PowerMEMS, Berkeley, USA, Nov. 29 - Dec. 1 2006, pp. 207-210.

[12] R. Xu and S. Kim, "Figures of merits of piezoelectric materials in energy harvesters," in PowerMEMS, Atlanta, Georgia, USA, Dec. 02 - 05 2012, pp. pp. 464-467.

[13] Y. Tsujiura, E. Suwa, H. Hida, K. Suenaga, K. Shibata, and I. Kanno, "Lead-free piezoelectric mems energy harvesters of stainless steel cantilevers," in Transducers, Barcelona, Spain, June 16-20 2013, pp. pp. $474-477$. 
[14] L. Minh, M. Hara, and H. Kuwano, "Miniaturised multi-beam (k,na)nbo3-based energy harvester," in PowerMEMS, Atlanta, Georgia, USA, Dec. 02 05 2012, pp. pp. 34-37.

[15] I. Kanno, T. Mino, S. Kuwajima, and T. Suzuki, "Piezoelectric properties of (k,na)nbo3 thin films deposited on (001)srruo3/pt/mgo substrates," IEEE Transactions on Ultrasonics, Ferroelectrics and Frequency Control, vol. 54, no. 12, pp. pp. 2562-2556, 2007.

[16] H. Mgbemere, R. Herber, and G. Schneider, "Investigation of the dielectric and piezoelectric properties of potassium sodium niobate ceramics close to the phase boundary at (k0.35na0.65)nbo3 and partial substitutions with lithium and antimony," J. Eur. Ceram. Soc., vol. 29, no. 15, pp. pp. 32733278, 2009.

[17] T. Yamashita, S. Takamatsu, T. Kobayashi, and T. Itoh, "Development of a fabrication process for a piezoelectric harvesting film using a reelto-reel continuous fier process," in Transducers, Barcelona, Spain, June 16-20 2013, pp. pp. 15611564.

[18] A. Erturk and D. Inman, Piezoelectric energy harvesting. New Delhi, India: Wiley, 2011.

[19] P. Glynne-Jones, S. Beeby, and N. White, "Towards a piezoelectric vibration-powered microgenerator," IEE Proc. Sci. Meas. Technol., vol. 148, no. 2, pp. $68-72$, 2001. [Online]. Available: http://dx.doi.org/10.1049/ip-smt:20010323

[20] Q. Tang and X. Li, "A wide-band piezoelectric energy-harvester for high-efficiency power generation at low frequencies," in IEEE Transducers, 16-20 June 2013, pp. 697-700. [Online]. Available: http://dx.doi.org/10.1109/ Transducers.2013.6626862

[21] Y. Jia and A. A. Seshia, "Comparison of five topologies of cantilever-based mems piezoelectric vibration energy harvesters," J. Phys. Conf. Ser., vol. 557, p. 5pp., 2014.

[22] S. Roundy and P. Wright, "A piezoelectric vibration based generator for wireless electronics," Smart Mater. Struct., vol. 13, no. 5, pp. 1131-1142, 2004.

[23] R. Andosca, T. G. McDonald, V. Genova, S. Rosenberg, J. Keating, C. Benedixen, and $\mathrm{J}$. Wu, "Experimental and theoretical studies on mems piezoelectric vibrational energy harvesters with mass loading," Sens. Actuators A, vol. 178, pp. 76-87, 2012. [Online]. Available: http: //dx.doi.org/10.1016/j.sna.2012.02.028

[24] Y. Jia and A. A. Seshia, "White noise responsiveness of an aln piezoelectric mems cantilever vibration energy harvester," J. Phys. Conf. Ser., vol. 557, p. 5pp, 2014.

[25] M. Stewart, P. Weaver, and M. Cain, "Charge redistribution in piezoelectric energy harvesters," Appl. Phys. Lett., vol. 100, no. 7, 2012.
[26] S. Arora, A. Arora, and P. George, "Design of mems based microcantilever using comsol multiphysics," Int. J. Appl. Eng. Res., vol. 7, no. 11, p. 3pp., 2012.

[27] W. Thomson, Theory of Vibration with Applications 5th Edition, M. Dahleh, Ed. New Jersey: Prentice-Hall, Inc., 1998.

[28] S. Senturia, Microsystem design. Dordrecht: Kluwer Academic Publishers, 2001.

[29] A. Erturk and D. Inman, "An experimentally validated bimorph cantilever model for piezoelectric energy harvesting from base excitations," Smart Mater. Struct., vol. 18, no. 2, p. 18pp, 2008.

[30] S.Roundy, "Energy scavenging for wireless sensor nodes with a focus on vibration to electricity conversion," Ph.D. dissertation, University of California, Berkeley, 2003.

[31] D. Zhu, S. Beeby, M. Tudor, and N. Narris, "A credit card sized self powered smart sensor node," Sens. Actuators A, p. 9pp, 2011.

[32] MicroGen. (2013) Bolt tm, $600 \mathrm{hz}$ micro-power generator. [Online]. Available: http://www.microgensystems.co/content/ MicroGen_R0600-datasheet_Jun2013.pdf 